\title{
The isotropic-cholesteric transition in liquid-crystalline gels
}

\author{
Robert A. Pelcovits \\ Department of Physics, Brown University, Providence, Rhode Island 02912 \\ Robert B. Meyer \\ The Martin Fisher School of Physics, Brandeis University, Waltham, Massachusetts 02254-9110
}

(Dated: November 9, 2018)

\begin{abstract}
In a nematic gel, the appearance of nematic order is accompanied by a spontaneous elongation of the gel parallel to the nematic director. If such a gel is made chiral, it has a tendency to form a cholesteric helical texture, in which local elongation of the gel parallel to the nematic director is suppressed due to the requirement of elastic compatibility. We show that a conical helix in which the director makes an oblique angle with respect to the helix axis serves as an energy minimizing compromise between the competing tendencies for elongation and twisting. We find the dependence of the helical cone angle and pitch on the strength of the chirality, and determine the change in sample shape at the isotropic to cholesteric phase transition.
\end{abstract}

PACS numbers: $64.70 . \mathrm{Md}, 61.30 . \mathrm{Vx}, 83.80 . \mathrm{Va}$

\section{INTRODUCTION}

A nematic gel is basically a polymer gel embedded in a nematic solvent [1]. To make these two systems compatible, the polymer of the gel is normally formed from monomer molecules that have a nematogen-like side chain attached to the reactive group that will form the polymer backbone. As in an ordinary polymer gel, the volume fraction of nematic solvent is of order $90 \%$. The fundamental interaction between the polymer chains and the nematic is an orientational coupling. Here we will assume that the flexible polymer backbone has a tendency to be aligned parallel to the nematic director. The primary implication of this coupling is that at the isotropic to nematic phase transition, a typical polymer coil in the gel transforms from a spherical object in the isotropic phase to a prolate ellipsoid aligned with its long axis parallel to the director in the nematic phase. Because the polymer coils are cross-linked in the gel, this shape transformation carries over from the individual polymer coils to the sample as a whole, leading to a spontaneous elongation of the entire sample parallel to the director.

This simple view of the transition assumes that the director is uniformly aligned throughout the sample. Experimentally this is found not to be the case in real materials, unless some step has been taken to bias the orientation of the director in the material [2,3]. In an unbiased sample, some kind of macroscopically isotropic, multidomain, nematic appears, the exact nature of which is not currently known. Since spontaneous elongation parallel to the director minimizes the free energy, this multi-domain state must combine some degree of local elongation, along with overall average isotropy. The problem is to see how to combine local elongations of many different orientations in an elastically compatible way.

In this paper, we study a problem that has elements of the general problem alluded to above. We examine the question of how a single domain of a cholesteric mesophase can form at the isotropic to cholesteric transition. In the ordinary cholesteric helix, the underlying nematic director is oriented perpendicular to the helix axis. If one tries to imagine local spontaneous elongations parallel to the director, there is a serious problem, since every quarter turn of the helix, the elongations would be in orthogonal directions, a clear impossibility. We suggest as a possible solution to this problem a compromise between the full twisting of the cholesteric helix, and the overall elongation of the sample. It is a conical helix, in which at every point in the helix, the director rotates by an angle $\theta$ toward the helix axis, and the sample can elongate parallel to the helix axis. A 90 degree rotation would change the cholesteric into a nematic, with the maximum elongation of the sample. We explore the possibility that some smaller rotation angle will minimize the free energy as a compromise between elongation and twisting.

\section{THEORY}

To study the isotropic-cholesteric transition we model the gel following the approach of Ref. [4]. We describe the orientational degrees of freedom using the symmetric-traceless nematic order parameter $Q_{i j}$, rather than a director $\mathbf{n}$, in order to describe the development of orientational order. The gel is modeled as an isotropic elastic medium; its free energy can be written in terms of the right Cauchy-Green tensor $u_{i j}$, or equivalently the left Cauchy-Green tensor $v_{i j}$. The latter tensor transforms like a rank-2 tensor under rotations in the target space of the elastic medium, 
i.e., the space defined by the locations of the mass points after a distortion. The former tensor, on the other hand, transforms like a rank-2 tensor under rotations in the reference space, defined by the locations of the mass points before a distortion occurs. The nematic order parameter tensor $Q_{i j}$ also transforms as a rank-2 tensor in the target space. Thus, in constructing the free energy, the coupling of the elastic degrees of freedom to the nematic degrees of freedom must involve contractions of $Q_{i j}$ with $v_{i j}$, rather than $u_{i j}$. The Cauchy-Green tensors are given by:

$$
\begin{aligned}
u_{i j} & =\frac{1}{2}\left(\partial_{i} u_{j}+\partial_{j} u_{i}+\partial_{i} u_{k} \partial_{j} u_{k}\right) \\
v_{i j} & =\frac{1}{2}\left(\partial_{i} u_{j}+\partial_{j} u_{i}+\partial_{k} u_{i} \partial_{k} u_{j}\right),
\end{aligned}
$$

where $\mathbf{u}(\mathbf{x})$ is the displacement vector associated with the elastic distortion, and $\partial_{i}$ denotes $\partial / \partial x_{i}$, with $x_{i}$ the $i$ th component of the position vector of the mass points in the reference space. Note that $\operatorname{Tr} \underline{\underline{u}}=\operatorname{Tr} \underline{\underline{v}}$ in general, while to linear order in $\mathbf{u}(\mathbf{x})$, the two elastic tensors are equal. We use the summation convention throughout, summing over repeated indices, which span the three-dimensional reference space.

The free energy density $f$ of the gel consists of an isotropic elastic term $f_{e l}(\underline{\underline{u}})$, a term $\left.f_{Q}^{\prime} \underline{\underline{Q}}\right)$ describing the nematic degrees of freedom (including gradient terms), and a term $f_{C}$ which couples the elastic and nematic degrees of freedom;

$$
f=f_{e l}(\underline{\underline{u}})+f_{Q}^{\prime}(\underline{\underline{Q}})+f_{C}
$$

The elastic energy $f_{e l}(\underline{\underline{u}})$ is given in by:

$$
f_{e l}(\underline{\underline{u}})=\frac{1}{2} \lambda(\operatorname{Tr} \underline{\underline{u}})^{2}+\mu \operatorname{Tr} \underline{\underline{u}}^{2},
$$

where $\lambda$ and $\mu$ are the Lamé coefficients. It suffices to consider only terms quadratic in $u_{i j}$.

The free energy $\left.f_{Q}^{\prime} \underline{\underline{Q}}\right)$ describing the nematic degrees of freedom is a sum of gradient [5] and bulk terms:

$$
\left.f_{Q}^{\prime} \underline{\underline{Q}}\right)=\frac{1}{4} K_{1}\left(\varepsilon_{i j k} \partial_{j} Q_{i k}+2 q_{o} Q_{i j}\right)^{2}+\frac{1}{4} K_{o}\left(\partial_{j} Q_{i j}\right)^{2}+\frac{1}{2} r_{Q}^{\prime} \underline{\underline{T r}} \underline{\underline{Q}}^{2}-w_{3} \operatorname{Tr} \underline{\underline{Q}}^{3}+w_{4}^{\prime}\left(\underline{\operatorname{Tr}}^{2}\right)^{2} .
$$

The elastic constants $K_{o}$ and $K_{1}$ are related to the Frank elastic constants and the nematic order parameter $S$ by:

$$
\begin{aligned}
& K_{22}=K_{1} S^{2} \\
& K_{11}=K_{33}=\frac{S^{2}}{2}\left(K_{o}+K_{1}\right),
\end{aligned}
$$

where $K_{11}, K_{22}$, and $K_{33}$ are the splay, twist and bend Frank constants respectively, $q_{o}$ is the cholesteric torsion of the mesogenic molecules, and $\varepsilon_{i j k}$ is the fully antisymmetric Levi-Civita tensor. The pitch of the ordinary cholesteric helix would be $2 \pi / q_{0}$. Terms of higher order in $Q_{i j}$ would be required to break the equality of the bend and splay elastic constants [6].

The simplest coupling between the elastic and nematic degrees of freedom is given by:

$$
f_{C}=-s \underline{\operatorname{Tr}} \underline{\underline{\operatorname{Tr}}} \underline{\underline{\underline{Q}}}-2 t \operatorname{Tr} \underline{\underline{\tilde{v}}} \underline{\underline{Q}}
$$

just as in the case of the $I-N$ transition. Here $\underline{\underline{\tilde{v}}}$ is the symmetric-traceless part of $v_{i j}: \tilde{v_{i j}}=v_{i j}-\frac{1}{3} \delta_{i j} v_{k k}$. In fact, the only difference between the energy $f$ used here and the corresponding one used in Ref. [4], is the presence in $f$ of the gradient terms proportional to $K_{o}$ and $K_{1}$, which are required to describe cholesteric ordering.

As in Ref. [4], it is convenient to complete the squares in the terms in $f$ involving Tr$\underline{\underline{u}}$ and $\underline{\underline{\tilde{v}}}$ and write:

$$
f=\frac{1}{2} B[\operatorname{Tr} \underline{\underline{u}}-(s / B) \operatorname{Tr} \underline{\underline{Q}}]^{2}+\mu \operatorname{Tr}[\underline{\underline{\tilde{v}}}-(t / \mu) \underline{\underline{Q}}]^{2}+f_{Q}
$$

where

$$
f_{Q}=\frac{1}{4} K_{1}\left(\varepsilon_{i j k} \partial_{j} Q_{i k}+2 q_{o} Q_{i j}\right)^{2}+\frac{1}{4} K_{o}\left(\partial_{j} Q_{i j}\right)^{2}+\frac{1}{2} r_{Q} \underline{\operatorname{Tr}} \underline{\underline{Q}}^{2}-w_{3} \underline{\operatorname{Tr}}^{3}+w_{4}\left(\underline{\operatorname{Tr}} \underline{\underline{Q}}^{2}\right)^{2},
$$

with $r_{Q}=r_{Q}^{\prime}-2\left(t^{2} / \mu\right)$ and $w_{4}=w_{4}^{\prime}-\left(s^{2} / 2 B\right)$. Here $B$ is the bulk modulus of the gel, given in terms of the Lamé coefficients by: $B=\lambda+\frac{2}{3} \mu$. 
We now consider the isotropic-cholesteric transition within mean-field theory, minimizing the free energy $f$ as a function of $\operatorname{Tr} \underline{\underline{u}}$, and the independent components of the symmetric-traceless tensors $\tilde{v}_{i j}$, and $Q_{i j}$. This minimization requires care on two scores. First, elastic compatibility must be ensured. The six quantities $\operatorname{Tr} \underline{u}, \tilde{v}_{i j}$ must obey compatibility equations consistent with the existence of an underlying single-valued continuous displacement field $\mathbf{u}(\mathbf{x})$. If we linearize the Cauchy-Green elastic tensors, compatibility requires [7]:

$$
\begin{aligned}
\partial_{y}^{2}\left(\tilde{v}_{x x}+\frac{1}{3} \operatorname{Tr} \underline{\underline{u}}\right)+\partial_{x}^{2}\left(\tilde{v}_{y y}+\frac{1}{3} \operatorname{Tr} \underline{\underline{u}}\right) & =2 \partial_{x} \partial_{y} \tilde{v}_{x y} \\
\partial_{z}^{2}\left(\tilde{v}_{y y}+\frac{1}{3} \operatorname{Tr} \underline{\underline{u}}\right)+\partial_{y}^{2}\left(\frac{1}{3} \operatorname{Tr} \underline{\underline{u}}-\tilde{v}_{x x}-\tilde{v}_{y y}\right) & =2 \partial_{y} \partial_{z} \tilde{v}_{y z} \\
\partial_{x}^{2}\left(\frac{1}{3} \operatorname{Tr} \underline{\underline{u}}-\tilde{v}_{x x}-\tilde{v}_{y y}\right)+\partial_{z}^{2}\left(\tilde{v}_{x x}+\frac{1}{3} \operatorname{Tr} \underline{\underline{u}}\right) & =2 \partial_{x} \partial_{z} \tilde{v}_{x z} \\
\partial_{y} \partial_{z}\left(\tilde{v}_{x x}+\frac{1}{3} \operatorname{Tr} \underline{\underline{u}}\right) & =\partial_{x}\left(-\partial_{x} \tilde{v}_{y z}+\partial_{y} \tilde{v}_{x z}+\partial_{z} \tilde{v}_{x y}\right) \\
\partial_{x} \partial_{z}\left(\tilde{v}_{y y}+\frac{1}{3} \operatorname{Tr} \underline{\underline{u}}\right) & =\partial_{y}\left(\partial_{x} \tilde{v}_{y z}-\partial_{y} \tilde{v}_{x z}+\partial_{z} \tilde{v}_{x y}\right) \\
\partial_{x} \partial_{y}\left(\frac{1}{3} \operatorname{Tr} \underline{\underline{u}}-\tilde{v}_{x x}-\tilde{v}_{y y}\right) & =\partial_{z}\left(-\partial_{x} \tilde{v}_{y z}+\partial_{y} \tilde{v}_{x z}-\partial_{z} \tilde{v}_{x y}\right) .
\end{aligned}
$$

Second, minimizing with respect to the nematic degrees of freedom requires an ansatz for the nature of the ordering. While gel "blue phases" could in principle form, with local elongations parallel to the double twist axes, here we consider the simpler possibility of a conical helical phase, which will allow the gel to elongate along the pitch axis (with small shears about this axis), yet still gain twist energy from the helical ordering. Minimization of the free energy will determine the optimal opening angle of the cone. A conical helix also forms in ordinary cholesterics in the presence of suitably strong (but not too strong) magnetic fields, if $K_{22}>K_{33}$ [8]. The qualitative similarity between a cholesteric in a magnetic field and a cholesteric gel is not surprising, given the form of the coupling proportional to $t$ appearing in $f_{C}$.

Assuming that the pitch axis in the ordered state lies along the $z$ axis, and the director makes an angle $\theta$ with the $x-y$ plane (so that $\theta=0$ corresponds to the ordinary helix), $Q_{i j}$ is given by:

$$
\underline{\underline{Q}}=S\left(\begin{array}{ccc}
\cos ^{2} \theta \cos ^{2} q z-\frac{1}{3} & \frac{1}{2} \cos ^{2} \theta \sin 2 q z & \frac{1}{2} \sin 2 \theta \cos q z \\
\frac{1}{2} \cos ^{2} \theta \sin 2 q z & \cos ^{2} \theta \sin ^{2} q z-\frac{1}{3} & \frac{1}{2} \sin 2 \theta \sin q z \\
\frac{1}{2} \sin 2 \theta \cos q z & \frac{1}{2} \sin 2 \theta \sin q z & \sin ^{2} \theta-\frac{1}{3}
\end{array}\right)
$$

where $S$ is the magnitude of the nematic order parameter, and $q$ is the torsion of the conical helix in the gel. These latter two quantities will be determined from the minimization procedure.

Within a mean-field theory treatment, there will be no dependence on the $x$ and $y$ coordinates, in which case the compatibility equations, Eqs. (11), (14), and (15) are trivially satisfied and Eqs. (12), (13) and (16) reduce respectively to:

$$
\begin{gathered}
\partial_{z}^{2}\left(\tilde{v}_{y y}+\frac{1}{3} \operatorname{Tr} \underline{\underline{u}}\right)=0 \\
\partial_{z}^{2}\left(\tilde{v}_{x x}+\frac{1}{3} \operatorname{Tr} \underline{\underline{u}}\right)=0 \\
\partial_{z}^{2} \tilde{v}_{x y}=0 .
\end{gathered}
$$

These latter compatibility equations represent integrable (i.e. holonomic) constraints on the components of the elastic tensor. We expect these components to be proportional to $\sin q z$ or $\cos q z$, and thus integrating these equations produces the constraints:

$$
\begin{aligned}
\tilde{v}_{x x}+\frac{1}{3} \operatorname{Tr} \underline{\underline{u}}-C & =0 \\
\tilde{v}_{y y}+\frac{1}{3} \operatorname{Tr} \underline{\underline{u}}-C & =0 \\
\tilde{v}_{x y} & =0,
\end{aligned}
$$

where $C$ is independent of $z$, and we have assumed cylindrical symmetry about the $z$ axis. While the component $\tilde{v}_{x y}$ could on the basis of Eq. (20) be nonzero, minimization of $f$ along with the form of $Q_{i j}$, Eq. (17), shows that the equilibrium value of $\tilde{v}_{x y}$ is in fact zero. 
For simplicity we consider from this point on the incompressible limit, $B \rightarrow \infty$ (in fact, real gels are nearly incompressible), in which case:

$$
\begin{aligned}
& \operatorname{Tr} \underline{\underline{u}}=0 \\
& \tilde{v}_{x x}=\tilde{v}_{y y}=C
\end{aligned}
$$

Using Eqs. (23)-(25) we minimize $f$ with respect to $C, \tilde{v}_{x z}$, and $\tilde{v}_{y z}$ and find:

$$
\begin{aligned}
2 C-(t / \mu)\left[Q_{x x}+Q_{y y}\right] & =0 \\
2 \mu\left[\tilde{v}_{x z}-(t / \mu) Q_{x z}\right] & =0 \\
2 \mu\left[\tilde{v}_{y z}-(t / \mu) Q_{y z}\right] & =0
\end{aligned}
$$

Using Eqs. (17), and (26)-(28) the free energy $f$ in the incompressible limit reduces to:

$$
\begin{aligned}
f=\frac{S^{2}}{2}\left\{\frac{1}{2}\left(K_{o}+K_{1}\right) q^{2} \sin ^{2} \theta \cos ^{2} \theta+\right. & \left.K_{1}\left(q \cos ^{2} \theta-q_{o}\right)^{2}+\frac{1}{3} K_{1} q_{o}^{2}+\frac{t^{2}}{\mu} \cos ^{4} \theta\right\} \\
& +\frac{1}{3} r_{Q} S^{2}-\frac{2}{9} w_{3} S^{3}+\frac{4}{9} w_{4} S^{4} .
\end{aligned}
$$

Minimizing $f$ with respect to $\theta$ we find:

$$
\frac{\partial f}{\partial \theta}=\sin 2 \theta\left\{K_{1} q\left(-q \cos ^{2} \theta+q_{o}\right)+\frac{1}{4}\left(K_{o}+K_{1}\right) q^{2} \cos 2 \theta-\left(t^{2} / \mu\right) \cos ^{2} \theta\right\}=0
$$

while minimizing with respect to $q$ yields:

$$
\frac{\partial f}{\partial q}=\frac{1}{2}\left(K_{o}-K_{1}\right) q \cos ^{2} \theta+K_{1} q_{o}-\frac{1}{2}\left(K_{o}+K_{1}\right) q=0 .
$$

Finally, minimizing with respect to $S$ yields:

$$
\begin{aligned}
\frac{\partial f}{\partial S}=S\left\{\frac{1}{2}\left(K_{o}+K_{1}\right) q^{2} \sin ^{2} \theta \cos ^{2} \theta\right. & \left.+K_{1}\left(q \cos ^{2} \theta-q_{o}\right)^{2}+\frac{2}{3} r_{Q}+\left(t^{2} / \mu\right) \cos ^{4} \theta\right\} \\
& -\frac{2}{3} w_{3} S^{2}+\frac{16}{9} w_{4} S^{3}=0 .
\end{aligned}
$$

Eq. (30) has the same form as the equation that determines the optimal value of $\theta$ for a cholesteric with magnetic susceptibility anisotropy $\Delta \chi$ in an effective magnetic field $H$, with

$$
\Delta \chi H^{2} \equiv 2\left(t^{2} S^{2} / \mu\right) \cos ^{2} \theta
$$

Using Eqs. (31) and (30), the values of $q$ and $\theta$ which minimize $f$ obey:

$$
\frac{1}{4}\left(K_{o}+K_{1}\right) q^{2}=\left(t^{2} / \mu\right) \cos ^{2} \theta
$$

In terms of the constant

$$
\beta=q_{0}\left(\frac{\mu}{t S}\right) \sqrt{\frac{K_{33}}{2 \mu}}
$$

and using Eq. (7), this relationship can be written as

$$
q=q_{0} \frac{\cos \theta}{\beta}
$$

Note that $q$ and $q_{0}$ have the same sign.

Since the ratio of $K_{33}$ to $K_{22}$ plays an important role, we define this ratio as $\gamma$ for the discussion below. 


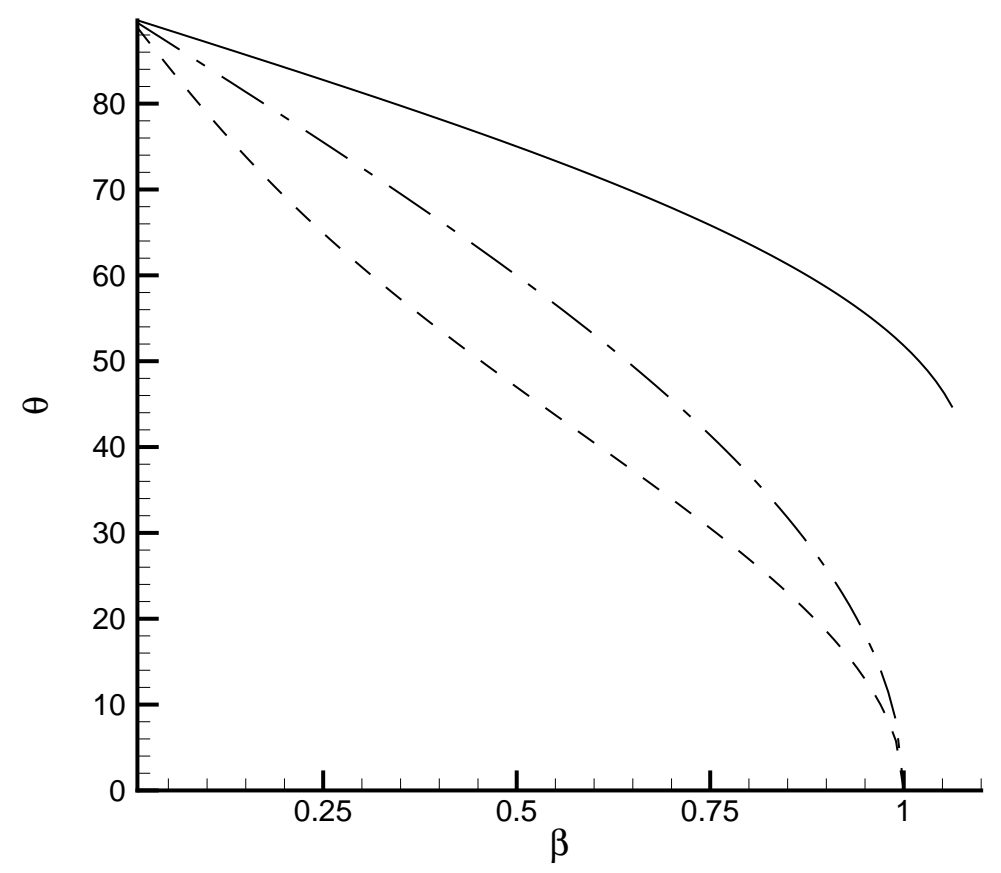

FIG. 1: The optimum angle $\theta$ of the conical helix as a function of the chirality parameter $\beta$, for three values of the elastic constant ratio $\gamma \equiv \frac{K_{33}}{K_{22}}=2,1$, and 0.5 , for the upper, middle, and lower curves, respectively.

Using Eq. (36) to eliminate $q$, we find that the free energy can be written as

$$
\begin{aligned}
f= & \frac{t^{2} S^{2}}{\mu \gamma}\left\{(1-\gamma) \cos ^{6} \theta+\frac{3}{2} \gamma \cos ^{4} \theta-2 \beta \cos ^{3} \theta+\frac{4}{3} \beta^{2}\right\} \\
& +\frac{1}{3} r_{Q} S^{2}-\frac{2}{9} w_{3} S^{3}+\frac{4}{9} w_{4} S^{4}
\end{aligned}
$$

and the solution to Eq. (30) obeys:

$$
(1-\gamma) \cos ^{3} \theta+\gamma \cos \theta-\beta=0,
$$

Note that $\beta$ is independent of $S$ (recall Eq. (7)), so this equation is independent of $S$ and the determination of the optimal angle $\theta$ is therefore independent of the value of $S$, as long as nematic order exists. However, as can be seen from Eq. (32), $S$ does depend on $\theta$.

The constant $\beta^{2}$ is a measure of the chiral bending energy in the conical helix, $K_{33} q_{0}^{2}$, measured in terms of the coupling energy of the nematic order to the strain field in the sample. To picture the trade-off between spontaneous elongation of the nematic and the chiral energy of the helix, start with $\beta=0$; for this value, the free energy is minimized for $\theta=\pi / 2$, a simple nematic, with no helix. For small $\beta, \cos \theta \approx \beta / \gamma$, or $\theta \approx \pi / 2-\beta / \gamma$, and the helix appears with $q=q_{0} \cos \theta / \beta \approx q_{0} / \gamma$. In other words, even the slightest chirality produces a helix, with a small cone angle $\phi \equiv \pi / 2-\theta \approx \beta / \gamma$, and a pitch $P \approx 2 \pi \gamma / q_{0}$ in which bend is the dominant curvature, as indicated by the factor $\gamma$. The factor $\gamma$ also determines how the cone angle grows, i.e., how $\theta$ decreases, with increasing $q_{0}$, or increasing $\beta$, as shown in Fig. 1.

Real materials have positive values of $\gamma$, on the order of 2. As seen in Fig. 1, for values of $\gamma$ less than about 1.5, as $\beta$ is increased to $1, \theta$ decreases smoothly to zero, restoring the ordinary cholesteric helix. For larger values of $\gamma$, as $\beta$ grows, $\theta$ decreases smoothly, and then at some value of $\beta$ greater than $1, \theta$ jumps discontinuously to zero. This is a trade-off between bend and twist energy in the helix; since the twist elastic constant is much less than the bend constant, the initially bend-dominated helix can lower its energy by this transformation. While the helix is either gradually or suddenly changing from bend to twist, its pitch is also evolving toward the value determined by pure twist, as indicated by Eq. (36). 

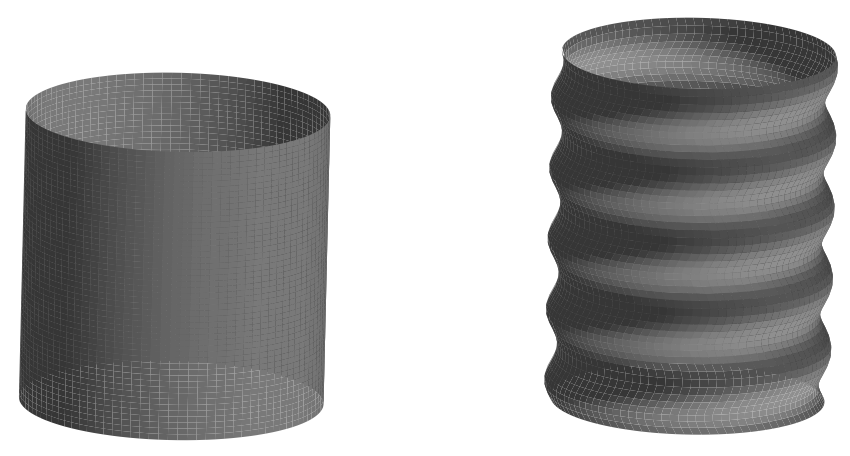

FIG. 2: The distortion of a cylindrical isotropic gel (left) after undergoing a transition to the conical helix phase (right) with a small cone angle.

To summarize, other than the scaling of the helix energy to the strain energy in determining the constant $\beta$, the geometric properties of the conical helix are determined by the nematic elastic energies. It is interesting to estimate the strength of the chirality needed to produce a significant cone angle. If we look at the case $\beta=0$, the spontaneous elongation of the sample on entering the nematic phase is of order $t S / \mu$. Let us set this to a value 2 . The other factor needed in evaluating $\beta$ for the conical helix is the length $\sqrt{K_{33} / 2 \mu}$. Estimating $K_{33}=10^{-11} \mathrm{~J} / \mathrm{m}$, and $\mu=10^{3} \mathrm{~J} / \mathrm{m}^{3}$, for a weak gel, this length is of order $0.1 \mu \mathrm{m}$. Using these numbers, to achieve a value of $\beta$ of about 0.5 , we need a helix pitch of a few tenths of a micrometer. This pitch is common for highly chiral nematics.

Now we turn to the nematic ordering and the elastic deformation of the sample due to the presence of the conical helix.

Because of the form of the coupling of the nematic order to the strain field, the energy of the conical helix only enters into a term in the free energy proportional to $S^{2}$, meaning that the chiral energy simply lowers the transition temperature of the isotropic to cholesteric phase transition, relative to what it would be for $q_{0}=0$. This effect is familiar for cholesterics in general.

More interesting is the effect of the conical helix on the shape of the sample. The elastic distortion induced by the phase transition can be determined from Eqs. (17), (23), (25) and (26), with the result that the strain tensor $\underline{\underline{v}}$ is given by:

$$
\underline{\underline{v}}=\left(\begin{array}{ccc}
\frac{t S}{2 \mu}\left(\cos ^{2} \theta-\frac{2}{3}\right) & 0 & \frac{t S}{2 \mu} \sin 2 \theta \cos q z \\
0 & \frac{t S}{2 \mu}\left(\cos ^{2} \theta-\frac{2}{3}\right) & \frac{t S}{2 \mu} \sin 2 \theta \sin q z \\
\frac{t S}{2 \mu} \sin 2 \theta \cos q z & \frac{t S}{2 \mu} \sin 2 \theta \sin q z & \frac{t S}{\mu}\left(\frac{2}{3}-\cos ^{2} \theta\right)
\end{array}\right)
$$

This equation can be easily solved for the displacement field $\mathbf{u}$, consistent with the compatibility requirements and incompressibility, with the results:

$$
\begin{aligned}
& u_{x}=\frac{t S}{2 \mu}\left(\cos ^{2} \theta-2 / 3\right) x+\frac{t S}{\mu} \sin 2 \theta \sin q z \\
& u_{y}=\frac{t S}{2 \mu}\left(\cos ^{2} \theta-2 / 3\right) y-\frac{t S}{\mu} \sin 2 \theta \cos q z \\
& u_{z}=-\frac{t S}{\mu}\left(\cos ^{2} \theta-2 / 3\right) z
\end{aligned}
$$

We illustrate this displacement field in Figs. 2 and 3 where we show the deformation of a cylindrical gel in the isotropic phase which then undergoes a transition to the cholesteric phase. As anticipated, for a small cone angle $\phi=\pi / 2-\theta$, such that $\cos ^{2} \theta<2 / 3$, the sample is elongated parallel to the helix axis, and the transverse shears produce ridges on its surface, as shown in Fig. 2. For large cone angle, i.e., for $\cos ^{2} \theta>2 / 3$, the helix is close to its twist form, and in fact the sample has shrunk along the helix axis, and expanded laterally, again with ridges, as shown in Fig. 3. This unexpected deformation represents a compromise in which again there is elongation parallel to the local director in the helix, but elastic compatibility demands equal elongation perpendicular to both the director 

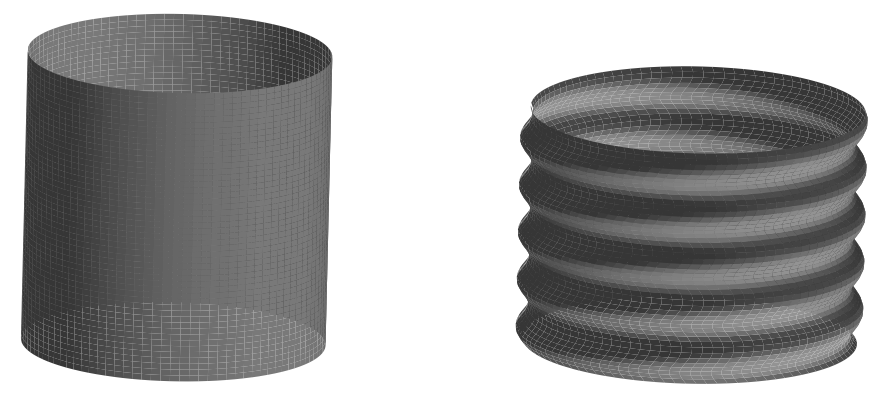

FIG. 3: The distortion of a cylindrical isotropic gel (left) after undergoing a transition to the conical helix phase (right) with a large cone angle.

and the helix axis, with shrinkage parallel to the helix axis to maintain constant volume. The possibility of this mode of deformation was first pointed out to us by Mark Warner [9].

\section{CONCLUSIONS}

In conclusion, we have found that the conical helix is a possible free energy minimizing texture of the cholesteric gel phase. It allows for a combination of local elongation of the system parallel to the nematic director, with some twisting due to the chirality of the material. It is fascinating to speculate on the existence of more complex textures that may offer an even better accommodation of the competing tendencies for spontaneous elongation and twist. We are currently looking into the possibility of periodic textures, similar to the blue phases, which may play this role.

\section{Acknowledgments}

We thank T. C. Lubensky and M. Warner for helpful discussions, and G. Loriot for assistance with Figs. 2 and 3. This work was supported by the National Science Foundation under Grant Nos. DMR-9873849, DMR-9974388, and DMR-0131573.

[1] M. Warner and E. M. Terentjev, Prog. Polym. Sci 21, 853 (1996).

[2] J. Küpfer and H. Finkelmann, Makromol. Chem. Rapid Commun. 12, 717 (1991).

[3] G. R. Mitchell, F. J. Davis, and W. Guo, Phys. Rev. Lett. 71, 2947 (1993).

[4] T. C. Lubensky, R. Mukhopadhyay, L. Radzihovsky, and X. Xing, Phy. Rev. E , (2002).

[5] D. C. Wright and N. D. Mermin, Rev. Mod. Phys. 61, 385 (1989).

[6] T. C. Lubensky, Phy. Rev. A 2, 2497 (1970).

[7] I. S. Sokolnikoff, Mathematical Theory of Elasticity (McGraw-Hill, New York, 1946).

[8] R. B. Meyer, App. Phys. Lett. 12, 281 (1968).

[9] Mark Warner (private communication). 